\title{
Are Nephrostomy and Ureteral Stent Necessary after Multi-Access Percutaneous Nephrolithotomy?
}

\author{
Seyed Mohammad Kazem Aghamir ${ }^{\mathrm{a}}$ Reza Heidari ${ }^{\mathrm{a}} \quad$ Seyedehsara Bayesh ${ }^{\mathrm{b}}$ \\ Alborz Salavati $^{\mathrm{a}}$ Reza Elmimehr ${ }^{\mathrm{a}}$

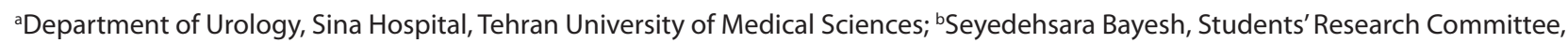 \\ Islamic Azad University, Tehran Medical Sciences Branch, Tehran, Iran
}

\author{
Key Words \\ Totally tubeless percutaneous nephrolithotomy • \\ Kidney stone $\bullet$ Renal stone
}

\begin{abstract}
Introduction: Totally tubeless percutaneous nephrolithotomy (PCNL) is a well-established technique used in single -access procedure nevertheless these results challenge the multi-access PCNL as a contra-indication to application of this technique. Aims: To compare complication rates and safety of use of this technique after multi-access PCNL. Methods: A Total of 130 patients with renal stones were enrolled in this cohort study. Totally tubeless PCNL with single access (Group A, $n=70$ ) and multi-access (Group B, $n=60$ ) were done and bleedings were assessed by measuring hemoglobin $(\mathrm{Hb})$ drop, blood transfusion rate and need for angioembolization besides leakage rates which were also recorded. Results: In the 130 patients who underwent totally tubeless PCNL, no leakage from puncture site nor angioembolization cases were identified. There was no significant statistical differences between 2 groups regarding Hb drop (1.91 in single access versus 2.20 in multi-access), transfusion rate and length of hospital stay ( $p>0.05)$. Stone-free rate was increased from $91 \%$ in single access to $97 \%$ in multi access by adding percutaneous access sites (from $91 \%$ in single access to $97 \%$ in multi-access). Conclusions: Multi-access totally
\end{abstract}

\section{KARGER}

Fax +4161306 1234

E-Mail karger@karger.com

www.karger.com
(C) 2019 The Author(s)

Open access

This article is licensed under the Creative Commons AttributionNonCommercial-NoDerivatives 4.0 International License (CC BYNC-ND) (http://www.karger.com/Services/OpenAccessLicense). Usage and distribution for commercial purposes as well as any distribution of modified material requires written permission. tubeless PCNL seems feasible and has comparable complication rates including $\mathrm{Hb}$ drop besides providing the advantage of higher stone-free rates using multi-access tracts.

(c) 2019 The Author(s)

Published by S. Karger AG, Basel

\section{Introduction}

Percutaneous nephrolithotomy (PCNL) is a minimally invasive surgical technique for removing stones of the renal pyelocalyceal system or upper ureter via a small percutaneous tract. Usually complex stones with diameters $>2 \mathrm{~cm}$ or shock wave-resistant lithiasis are managed using this technique [1-3]. Bellman et al. [4] in 1997 reported the first use of tubeless PCNL. In this procedure the nephrostomy tube (NT) as a final stage of PCNL was replaced by an internal stent $[5,6]$. Tubeless PCNL and standard PCNL have been compared by 4 systematic reviews. Meta-analysis and decrease in length of hospital stay, analgesia requirement, risk of bleeding, and postoperative morbidity were shown as advantages of tubeless PCNL method [7-10]. Recent studies in the past decade showed that totally tubeless PCNL without inserting ureter stents and nephrostomy catheter for those without any residual stones is gaining its place as a safe technique [11-14]. This study was designed to compare complica- 
Table 1. Patient's clinical characteristics, quantitative and qualitative variables

\begin{tabular}{|c|c|c|c|c|c|c|c|}
\hline Age, year & $45 \pm 11.07$ & $43.27 \pm 19.94$ & 0.58 & $41.36 \pm 20.85$ & 0.26 & $42.20 \pm 20.05$ & 0.32 \\
\hline Female, $\mathrm{n}$ & $45(64 \%)$ & $11(36.7 \%)$ & & $13(43.3 \%)$ & & $24(40 \%)$ & 0.10 \\
\hline Stone burden, $\mathrm{cm}^{3}$ & $2.81 \pm 0.59$ & $3.25 \pm 0.52$ & 0.001 & $3.22 \pm 0.66$ & 0.003 & $3.23 \pm 0.59$ & $<0.001$ \\
\hline Pre Hb, mg/dl & $14.34 \pm 1.34$ & $14.04 \pm 1.33$ & 0.31 & $13.87 \pm 1.58$ & 0.13 & $13.94 \pm 1.45$ & 0.10 \\
\hline Transfusion rate, $\%$ & $5(7 \%)$ & $1(9.1 \%)$ & & $0(0 \%)$ & & $1(4 \%)$ & 0.10 \\
\hline Angioembolization & 0 & 0 & & 0 & & 0 & \\
\hline Hospital stay, day & $3.1 \pm 0.7$ & $3.54 \pm 0.68$ & $>0.05$ & $3.42 \pm 0.85$ & $>0.05$ & $3.48 \pm 0.77$ & $>0.05$ \\
\hline Stone-free rate, $\%$ & 91 & 96 & & 98 & & 97 & 0.6 \\
\hline Leak from puncture, $\mathrm{ml}$ & 0 & 0 & & 0 & & 0 & \\
\hline
\end{tabular}

tion rates and safety of use of this technique after multiple access PCNL. To our knowledge, this is the first report of multi-access totally tubeless PCNL.

\section{Methods}

This historic cohort study was carried out in Tehran University of Medical Sciences, between 2014 and 2016 in Sina Hospital, Tehran, Iran. Our study was approved by Urology Research Center of Tehran University of Medical Sciences and study protocol conformed to the ethical guidelines of the 1975 declaration of Helsinki. Written informed consent was obtained from all individual participants included in this study. A Total of 130 patients with renal stones were enrolled in this cohort study. Totally tubeless PCNL were carried out in single access (Group A, $\mathrm{n}=70$ ) or multi accesses (Group B, n $=60$ ) and bleedings were assessed by measuring hemoglobin $(\mathrm{Hb})$ drop in first 24 post-operative hours, blood transfusion rates and need for performing angioembolization; besides leakage rates were also reported. Patients with extracorporeal shock wave lithotripsy failure, large renal stones ( $>25$ $\mathrm{mm}$ in pelvis or $>20 \mathrm{~mm}$ in lower calyx), or stones in diverticulum or closed calyx which were nonhomogeneous ranging from single calyx stone to complete staghorn stones who had normal coagulation tests with ASA (American Society of Anesthesiology) functional class I-III, $\mathrm{Hb}$ concentration above $10 \mathrm{mg} / \mathrm{dl}$ and negative urine culture where included. The exclusion criteria were intra-operative bleeding which caused persistent unclear vision during nephroscopy, perforation of the collecting system and unstable vital signs (persistent systolic pressure under $100 \mathrm{mmHg}$ ). General anesthesia in prone position and fluoroscopy was used to obtain the nephrostomy tract and ballistic lithotripsy was used. Finally, a C-arm multidirectional fluoroscopy was used for the assessment of probable stone remnants over $4 \mathrm{~mm}$ as flexible endoscopes were not available at the time of study design, therefore, cases managed with flexible nephroscopy were excluded to keep the sample uniform. In presence of significant remnant in patients without exclusion criteria, the second or third access was obtained.
After satisfactory stone removal, ureteral stent and Amplatz $(16 \mathrm{~cm} ; 30 \mathrm{~F})$ sheath were withdrawn, and compressive dressing was applied. In absence of wound leakage, the Foley catheter was removed after 12 to 24 hours. Hb serum level was measured preoperatively and 6 hours after the procedure and if there was not a significant drop, it would be measured daily until hematuria was resolved. Hemodynamically stable patients with controllable pain with painkillers and no gross hematuria were considered suitable for discharge after 3-5 days. About a week after discharge, patients were followed-up by radiography of the kidneys, ureters and bladder and renal ultrasonography to rule out residual stones and urinoma. Criteria for post-operative blood transfusion were unstable vital signs and $\mathrm{Hb}<10 \mathrm{mg} / \mathrm{dl}$. Angioembolization criteria were persistent bleeding that needed transfusion of more than 2 units during 24 hours or on re-admission due to persistent bleeding after discharge $[7,8]$. The data on stone burden, pre- and postoperative $\mathrm{Hb}$, transfusion rate, angioembolization, leakage from tract, duration of hospital stay and stone-free rate were collected. Statistical analysis was performed by SPSS software (ver.22) using multivariate analysis and Mann-Whitney test for continuous variables and chi square test for categorical variables. $\mathrm{P}$ values < 0.05 were considered statistically significant.

\section{Results}

In this cohort study, single-access totally tubeless PCNL compared with multi-access (3 or 4 ) in respect to demographic data, hemoglobin drop, stone-free rate and clinical characteristics (table 1). There were no significant differences between the age and gender of patients $(\mathrm{p}>0.05)$.

The mean stone burden was $2.81 \pm 0.59,3.25 \pm 0.52$ ), $3.22 \pm 0.66$, and $3.23 \pm 0.59 \mathrm{~cm}^{3}$ in single-, $3-, 4$ - and overall multi-access group (Group B), respectively. The results showed that there were significant differences between stone burden in single- and multi-access sites $(\mathrm{p}<0.001)$. 
Hospital stay was longer in multi-access group compared to single-access group and there was no significant relation $(\mathrm{p}>0.05)$. The $\mathrm{Hb}$ drop $(1.91 \mathrm{mg} / \mathrm{dl}$ in single access versus $2.20 \mathrm{mg} / \mathrm{dl}$ in multi-access) and transfusion rates were not significantly different in 2 groups ( $\mathrm{p}$ $=0.32$ ). Leakage from puncture site didn't occur more than 48 hours and no one needed ureteral double $\mathrm{J}$ stent. There was no case of angioembolization. Stone-free rate was 91, 96 and 98\% in single-, 3- and 4-access group, respectively. The results showed that the stone-free rate was increased by adding access sites.

\section{Discussion}

Despite the advantages of PCNL and its evolution since introduction by Fernström et al. [15] in 1976, several complications such as injury to renal blood vessels and hemorrhage, urine leakage are major complications inflicting some patients. Nephrostomy catheter which was routinely placed in the collecting system to act as a continuous access for second stage procedure, urinary drainage and tamponade bleeding from the tract between the skin and the kidney, had been a standard but had adverse effects such as pain and need for extra analgesic and prolonged hospital stay and excessive wound dressing $[8,16]$. Furthermore, in recent years, efforts have been made to change PCNL technique to reduce nephrostomy tube complications by using an externalized ureteral stent or double $\mathbf{J}$ stent $[2,17]$. In 1984, Wickham et al. [18] introduced tubeless PCNL for the first time. Then in 1997, Bellman et al. [4] discussed about the advantages of tubeless PCNL in comparison to standard PCNL and they reported lower length of hospital stay, time to return to daily activities, analgesia requirements and cost. Limb et al. [19] presented tubeless PCNL as a useful and safe method and Agrawal et al. [20] compared standard with tubeless PCNL and explained post-operative advantages of tubeless PCNL including reduced length of hospital stay, pain and urinary leakage.

Totally tubeless PCNL is a method of choice for the treatment of urinary stone disease in some centers $[12$, 21]. This technique uses neither a nephrostomy catheter nor a ureteral stent $[2,11-13,17,22,23]$. The advantages of totally tubeless PCNL include omitting of stent-related morbidity (flank pain, dysuria) [24], besides decreased length of hospital stay and need for analgesics [12, 25, 26]. Crook et al. [12] in 2008 reported totally tubeless PCNL as a safe method for selected patients without severe hematuria and complete stone clearance.

Multi-Access Percutaneous

Nephrolithotomy
Karami et al. [13] compared a total of 60 patients which divided in 2 equal groups who either underwent PCNL or totally tubeless PCNL. They reported post-operative discomfort reduced by removing nephrostomy tube and ureteral catheter. Crook et al. [12] and Yun et al. [24] also emphasized on advantages of totally tubeless PCNL. In a retrospective study performed by Istanbulluoglu et al. [14], 176 patients were divided into 3 groups of totally tubeless PCNL $(n=43)$, tubeless PCNL $(n=41)$ and standard PCNL $(\mathrm{n}=92)$. The groups were compared and no significant differences were found in mean stone burden, operation time, $\mathrm{Hb}$ drop and transfusion requirement rate. Time of hospital stay and analgesic requirement were significantly higher in standard PCNL compared with the other groups.

To our knowledge this is the first study to compare $\mathrm{Hb}$ drop and transfusion rate in single-access totally tubeless PCNL, with multiple-access (3 and 4). In this study, average $\mathrm{Hb}$ drop in single-access totally tubeless PCNL compared with 3 - and 4-access was not significantly different $(1.91 \pm 1.60$ vs. $2.62 \pm 2.14$ vs. $1.87 \pm 1.27 \mathrm{mg} / \mathrm{dl}$, $\mathrm{p}=0.176)$. No patient needed angioembolization in either groups and blood transfusion rates had no statistical difference $5(7 \%)$ versus $1(9.1 \%)$ and $0(0 \%)$.

In a randomized controlled trial compared standard PCNL and totally tubeless PCNL on 50 patients with renal stones, Crook et al. [11] reported that there were no significant intergroup differences in hemorrhage and blood transfusion values. In Korea, Yun et al. [24] compared outcomes between standard PCNL and totally tubeless PCNL on 57 patients with renal stones and reported blood loss was not significantly different between the 2 groups. Istanbulluoglu et al. [14] performed tubeless, totally tubeless, and standard PCNL on 176 patients with renal stones in a retrospective study and reported that no significant differences were found in mean transfusion rates and $\mathrm{Hb}$ level change between the groups.

Our results show that surgeon can safely proceed with obtaining extra accesses in case of high stone burden or any condition that might require multi-access PCNL and yet the total tubeless option can be used in these patients with comparable results to single-access totally tubeless PCNL patients.

\section{Conclusions}

Totally tubeless technique is a variant of PCNL that can be used in multi-access PCNL with comparable results to single-access totally tubeless PCNL regarding 
major complications including $\mathrm{Hb}$ drop and leakage. Besides, the surgeon can improve stone-free rates via more accesses without increasing risk of complications. Further studies with large sample size is warranted.

\section{Acknowledgments}

We thank the physicians and all participations of Sina hHospital that helped us in this study.

\section{References}

1 Lahme S, Bichler KH, Strohmaier WL, Götz $\mathrm{T}$ : Minimally invasive PCNL in patients with renal pelvic and calyceal stones. Eur Urol 2001;40:619-624.

2 Shah HN, Kausik VB, Hegde SS, Shah JN, Bansal MB: Tubeless percutaneous nephrolithotomy: a prospective feasibility study and review of previous reports. BJU Int 2005; 96:879-883.

3 Matlaga BR, Kim SC, Lingeman JE: Improving outcomes of percutaneous nephrolithotomy: access. EAU Update Series 2005;3:3743.

Bellman GC, Davidoff R, Candela J, Ger-

4 spach J, Kurtz S, Stout L: Tubeless percutaneous renal surgery. J Urol 1997;157:15781582.

5 Lojanapiwat B, Soonthornphan S, Wudhikarn S: Tubeless percutaneous nephrolithotomy in selected patients. J Endourol 2001;15:711713.

6 Goh M, Wolf JS Jr: Almost totally tubeless percutaneous nephrolithotomy: further evolution of the technique. J Endourol 1999;13: 177-180.

7 Zhong Q, Zheng C, Mo J, Piao Y, Zhou Y, Jiang Q: Total tubeless versus standard percutaneous nephrolithotomy: a meta-analysis. J Endourol 2013;27:420-426.

8 Wang J, Zhao C, Zhang C, Fan X, Lin Y, Jiang Q: Tubeless vs standard percutaneous nephrolithotomy: a meta $\square$ analysis. BJU Int 2012;109:918-924.

9 Amer T, Ahmed K, Bultitude M, Khan S, Kumar P, De Rosa A, Khan MS, Hegarty N: Standard versus tubeless percutaneous nephrolithotomy: a systematic review. Urol Int 2012;88:373-382.
10 Yuan H, Zheng S, Liu L, Han P, Wang J, Wei Q: The efficacy and safety of tubeless percutaneous nephrolithotomy: a systematic review and meta-analysis. Urol Res 2011; 39:401-410.

11 Crook T, Lockyer C, Keoghane S, Walmsley B: A randomized controlled trial of nephrostomy placement versus tubeless percutaneous nephrolithotomy. J Urol 2008;18: 612-614.

12 Crook TJ, Lockyer C, Keoghane SR, Walmsley BH: Totally tubeless percutaneous nephrolithotomy. J Endourol 2008;22:267272.

13 Karami H, Gholamrezaie HR: Totally tubeless percutaneous nephrolithotomy in selected patients. J Endourol 2004; 18:475-476.

14 Istanbulluoglu MO, Cicek T, Ozturk B, Gonen M, Ozkardes H: Percutaneous nephrolithotomy: nephrostomy or tubeless or totally tubeless? Urology 2010;75:1043-1046.

15 Fernström I, Johansson B: Percutaneous pyelolithotomy. A new extraction technique. Scand J Urol Nephrol 1975;10:257-259.

16 Desai MR, Kukreja RA, Desai MM, Mhaskar SS, Wani KA, Patel SH, Bapat SD: A prospective randomized comparison of type of nephrostomy drainage following percutaneous nephrostolithotomy: large bore versus small bore versus tubeless. J Urol 2004; 172:565-567.

17 Mouracade P, Spie R, Lang H, Jacqmin D, Saussine C: Tubeless percutaneous nephrolithotomy: what about replacing the Double-J stent with a ureteral catheter? J Endourol 2008;22:273-276.
18 Wickham J, Miller R, Kellett M, Payne S: Percutaneous nephrolithotomy: one stage or two? Br J Urol 1984;56:582-585.

19 Limb J, Bellman GC: Tubeless percutaneous renal surgery: review of first 112 patients. Urology 2002;59:527-531.

20 Agrawal MS, Agrawal M, Gupta A, Bansal S, Yadav A, Goyal J: A randomized comparison of tubeless and standard percutaneous nephrolithotomy. J Endourol 2008;22:439-442.

21 Aghamir SM, Salavati A, Aloosh M, Farahmand H, Meysamie A, Pourmand G: Feasibility of totally tubeless percutaneous nephrolithotomy under the age of 14 years: a randomized clinical trial. J Endourol 2012; 26:621-624.

22 Rana AM, Bhojwani JP, Junejo NN, Das Bhagia SD: Tubeless PCNL with patient in supine position: procedure for all seasons? with comprehensive technique. Urology 2008;71:581-585.

23 Tefekli A, Altunrende F, Tepeler K, Tas A, Aydin S, Muslumanoglu AY: Tubeless percutaneous nephrolithotomy in selected patients: a prospective randomized comparison. Int Urol Nephrol 2007;39:57-63.

24 Yun SI, Lee YH, Kim JS, Cho SR, Kim BS, Kwon JB: Comparative study between standard and totally tubeless percutaneous nephrolithotomy. Korean J Urol 2012;53:785-789.

25 Aghamir SMK, Modaresi SS, Aloosh M, Tajik A: Totally tubeless percutaneous nephrolithotomy for upper pole renal stone using subcostal access. J Endourol 2011;25:583-586.

26 Aghamir S, Hosseini S, Gooran S: Totally tubeless percutaneous nephrolithotomy. J Endourol 2004;18:647-648. 